\title{
ciRS-7 Promotes the Proliferation and Migration of Papillary Thyroid Cancer by Negatively Regulating the miR-7/Epidermal Growth Factor Receptor Axis
}

\author{
Jun-ya Han $\mathbb{D}^{1,2,3}$ Si Guo, ${ }^{4,5}$ Na Wei, ${ }^{1,2}$ Rui Xue, ${ }^{6}$ Wencai Li, ${ }^{1}$ Gang Dong, ${ }^{7}$ Jianhua Li, ${ }^{8}$ \\ Xiangyu Tian, ${ }^{1,2}$ Chao Chen, ${ }^{1,2}$ Sen Qiu, ${ }^{1,2}$ Tong Wang, ${ }^{1,2}$ Qiankun Xiao, ${ }^{1,2}$ \\ Chenguang Liu $\left(\mathbb{1},,^{1,3}\right.$ Jingjing Xu $\left(\mathbb{1},{ }^{1,2,3}\right.$ and Kui-sheng Chen $\mathbb{1}^{1,2,3}$ \\ ${ }^{1}$ Department of Pathology, The First Affiliated Hospital of Zhengzhou University, No. 1 Jian She Dong Avenue, Zhengzhou, China \\ ${ }^{2}$ Henan Key Laboratory of Tumor Pathology, No. 1 Jian She Dong Avenue, Zhengzhou, China \\ ${ }^{3}$ Academy of Medical Sciences, Zhengzhou University, No. 100 Ke Xue Avenue, Zhengzhou, China \\ ${ }^{4}$ Department of Medical Laboratory, Henan Provincial People's Hospital, Zhengzhou, Henan, China \\ ${ }^{5}$ Department of Medical Laboratory of Central China Fuwai Hospital, Central China Fuwai Hospital of Zhengzhou University, \\ Zhengzhou, Henan, China \\ ${ }^{6}$ Medical Research Center, The First Affiliated Hospital of Zhengzhou University, No. 1 Jian She Dong Avenue, Zhengzhou, China \\ ${ }^{7}$ Department of Ultrasound, The First Affiliated Hospital of Zhengzhou University, No. 1 Jian She Dong Avenue, Zhengzhou, China \\ ${ }^{8}$ Thyroid Surgery Department of the First Affiliated Hospital of Zhengzhou University, No. 1 Jian She Dong Avenue, \\ Zhengzhou, China
}

Correspondence should be addressed to Jingjing Xu; happyxujingjing@163.com and Kui-sheng Chen; chenksh2002@163.com

Received 18 September 2019; Revised 17 January 2020; Accepted 11 March 2020; Published 22 June 2020

Academic Editor: Guangcun Huang

Copyright (c) 2020 Jun-ya Han et al. This is an open access article distributed under the Creative Commons Attribution License, which permits unrestricted use, distribution, and reproduction in any medium, provided the original work is properly cited.

Purpose. The incidence of papillary thyroid cancer (PTC) is increasing, and traditional diagnostic methods are unsatisfactory. Therefore, identifying novel prognostic markers is very important. ciRS-7 has been found to play an important role in many cancers, but its role in PTC has not been reported. This study was performed to evaluate the biological role and mechanism of ciRS-7 in PTC. Material and Methods. The expression of ciRS-7 in PTC tissues and the matched adjacent tissues was determined by quantitative reverse transcription polymerase chain reaction (qRT-PCR). The PTC cell lines (TPC-1 and BCPAP) were used to evaluate the role of ciRS-7. ciRS-7-siRNA and overexpression plasmid were constructed and transfected into PTC cells. A CCK-8 assay and colony formation assay were performed to explore the effects of ciRS-7 on cell proliferation. Annexin V/PI staining and FACS detection were used to detect cell apoptosis. Wound healing assay was performed to detect cell migration. A transwell assay was conducted to explore the effects of ciRS-7 on invasion and migration. Western blotting was performed to evaluate protein expression. The luciferase reporter system was used to determine the underlying mechanism of miR-7. Result. ciRS-7 was highly expressed in PTC tissues and cell lines compared with the corresponding controls. In vitro study showed that ciRS-7 silencing suppressed proliferation, migration, and invasion of TPC-1 and BCPAP. Mechanistically, the effects of ciRS-7 on invasion and migration may be related to epithelial-mesenchymal transition (EMT). ciRS-7 silencing could attenuate effects on PTC cells induced by miR-7 knockdown. Epidermal growth factor receptor (EGFR), which was demonstrated to be a target of miR-7, decreased significantly in ciRS-7-siRNA PTC cells. Overexpression of EGFR also attenuated effects of PTC cells induced by silencing ciRS-7. Conclusion. ciRS-7 was significantly upregulated in PTC tissues, and it promoted the progression of PTC by regulating the miR-7/EGFR axis. ciRS-7 is a promising prognostic biomarker and therapeutic target in PTC. 


\section{Introduction}

Papillary thyroid cancer (PTC) is the most prevalent thyroid malignancy, accounting for $80-85 \%$ of all thyroid cancers [1]. The incidence of PTC has steadily and rapidly increased over the past 40 years. Particularly, in the past five years, the incidence has increased faster than that of any other type of cancer. Although PTC has a good prognosis, 30\% of patients may develop persistent disease or relapse. Somatic BRAFV600E mutation is used for diagnosing PTC, but its prognostic effect remains controversial [2-4]. Most PTCs are surgically removed and treated with adjuvant radioactive iodine. Nevertheless, a fraction of PTCs progresses to metastatic disease and/or does not respond to adjuvant radioactive iodine therapy; the prognosis of these cases is poor, with a 10 -year survival rate of $10 \%$ [5-7]. New prognostic markers useful for optimizing therapy and long-time follow-up care must be identified. Circular RNAs are a class of RNA molecules without $5^{\prime}$ and $3^{\prime}$ ends, cap structure, or poly-A tail structure. Because of their circular configuration, they are protected from degradation by RNases [8]. This is advantageous compared to the use of linear RNA as markers for diagnosis and prognosis [9]. Recent studies indicated that circRNAs play a critical regulatory function in multiple physiological and pathological processes. For example, circRNA_ 102958 sponges miR-585 to promote the tumorigenesis of colorectal cancer [10]. In nucleus pulposus tissues of intervertebral disc degeneration, circRNA_104670 sponges miR$17-3 p$ to regulate matrix metalloproteinase-2 [11]. In particular, the role of circRNAs as miRNA "sponges" in tumorigenesis and development has been widely investigated [9]. Unfortunately, although emerging evidences indicated that circRNAs play a critical regulatory function in proliferation and invasion of cancer cells, the study about functions of circRNA in PTC has just begun. Moreover, whether circRNAs in PTC sponge to miRNAs and regulate the expression level of downstream genes is also unclear.

Few evidence about functions and mechanisms of circRNAs exists at present.

ciRS-7, also named as cerebellar degeneration-related protein 1 antisense RNA (CDR1as) or CDR1NAT, comprises 1500 nucleotides and is predominantly present in the brain of humans and mice [12]. It plays a significant role in the diagnosis, prognosis, and treatment of various malignant tumors, as it targets microRNA-7 (miR-7) in multiple tumor types [13]. In this study, we found that ciRS-7 expression was significantly elevated in PTC tissues and cell lines compared with their normal controls. Upregulation of ciRS-7 is closely associated with poor prognoses. Mechanically, we demonstrated that ciRS-7 could act as a sponge of miR-7 to upregulate the level of EGFR and trigger PTC proliferation, invasion, and migration. Our data revealed that ciRS-7 overexpression promotes PTC progression via the miR-7/EGFR axis. ciRS-7 may be a prognostic marker and therapeutic target for PTC.

\section{Materials and Methods}

2.1. Human Tissues. A total of 17 patients at the First Affiliated Hospital of Zhengzhou University (Zhengzhou, China) with PTC confirmed pathologically were enrolled in this study. Fresh thyroid carcinoma tissues and adjacent normal thyroid tissues located more than $3 \mathrm{~cm}$ away from the cancer site were collected and immediately frozen in liquid nitrogen. No patient had been administered therapy. The research protocol was approved by the Ethics Committees of the First Affiliated Hospital of Zhengzhou University. All patients provided informed written consent for all procedures. The clinicopathological parameters were archived from the medical record.

2.2. Cell Culture and Transfection. Human PTC cell TPC-1 and BCPAP and human thyroid follicular epithelial cell line Nthy-ori 3-1 were acquired from American Type Culture Collection (Manassas, VA, USA). They were cultured in Roswell Park Memorial Institute (RPMI) 1640 medium containing 10\% fetal bovine serum (Gibco, Grand Island, NY, USA), $100 \mathrm{U} / \mathrm{mL}$ penicillin, and $100 \mu \mathrm{L} / \mathrm{mL}$ streptomycin. They were incubated in a humidified chamber in an atmosphere of $5 \% \mathrm{CO}_{2}$ at $37^{\circ} \mathrm{C}$. ciRS-7-siRNA, ciRS-overexpressed plasmid, miR-7 mimic, miR-7 inhibitor, EGFR-overexpressed plasmid, and the empty vectors were obtained from Genema (Shanghai, China). Cell transfections were performed using Lipofectamine 2000 reagent (Invitrogen) according to the manufacturer's instructions.

2.3. RNA Extraction and Quantitative qRT-PCR. The total RNA from tissues or cells was isolated using TRIzol reagent (Beyotime, Shanghai, China) according to the manufacturer's instructions. Complementary DNA (cDNA) was generated by reverse transcription using a PrimeScript ${ }^{\mathrm{TM}} \mathrm{RT}$ reagent kit (Takara, Shiga, Japan). Quantitative PCR was performed with TB Green Premix Ex Taq ${ }^{\mathrm{TM}}$ II (TaKaRa) on an ABI Prism 7900 sequencer (Applied Biosystems, Foster City, CA, USA). U6 was used to normalize the level of microRNA-7 expression, while GAPDH was used as an internal control for the determination ciRS-7. The data were analyzed using the $2^{-\Delta \Delta C T}$ method. Primer sequences were synthesized by SanYa (Shanghai, China) as follows: ciRS-7, R (reverse): $5^{\prime}$-TGTATCCAGAGTTACTTCCAGTGT-3', F (forward): $5^{\prime}$-TCAGCAGTTTCATCTTCTTCTTCA-3'; microRNA-7, RT (reverse transcription): $5^{\prime}$-GTCGTATCC AGTGCAGGGTCCGAGGTATTCGCACTGGAT CGAC AACAAC-3', R (reverse): 5' -CGCGCGTGGAAGACTA GTGATTTT- ${ }^{\prime}$, $\quad$ F (forward): $5^{\prime}$-AGTGCAGGGTCCGA GGTATT-3'.

2.4. Western Blot Assay. All the cells and tissues were lysed in RIPA buffer containing protease and phosphatase inhibitors. Proteins were separated by $10 \%$ SDS-polyacrylamide gels and transferred to polyvinylidene difluoride membrane (Bio-Rad, Hercules, CA, USA). Membranes were incubated at $4^{\circ} \mathrm{C}$ with anti-EGFR $(1: 1000$; Abcam, Cambridge, UK), anti-E-cadherin (1:1000; Abcam), anti-vimentin $(1: 1000$; Abcam), and anti-GAPDH (1:5000; Abcam) antibodies overnight. Subsequently, membranes were incubated with HRP-conjugated secondary antibodies. Protein expression levels were visualized using electrochemiluminescence substrates (Pierce, Rockford, IL, USA). 


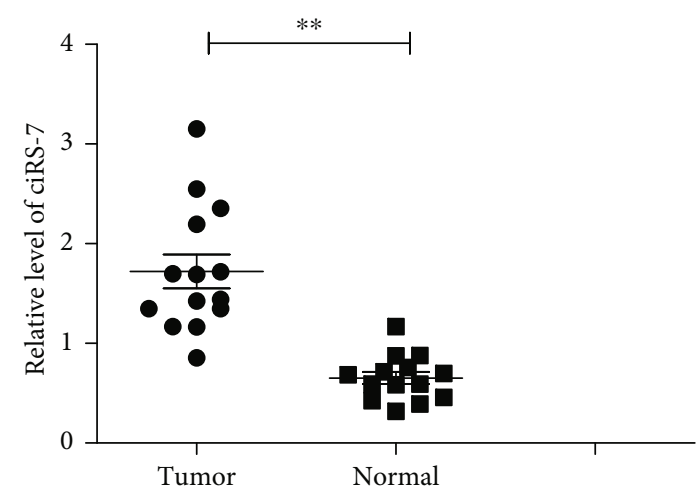

(a)

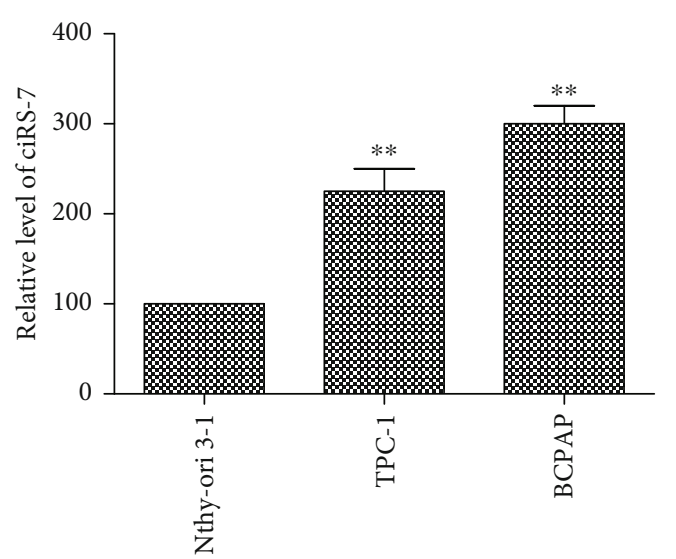

(b)

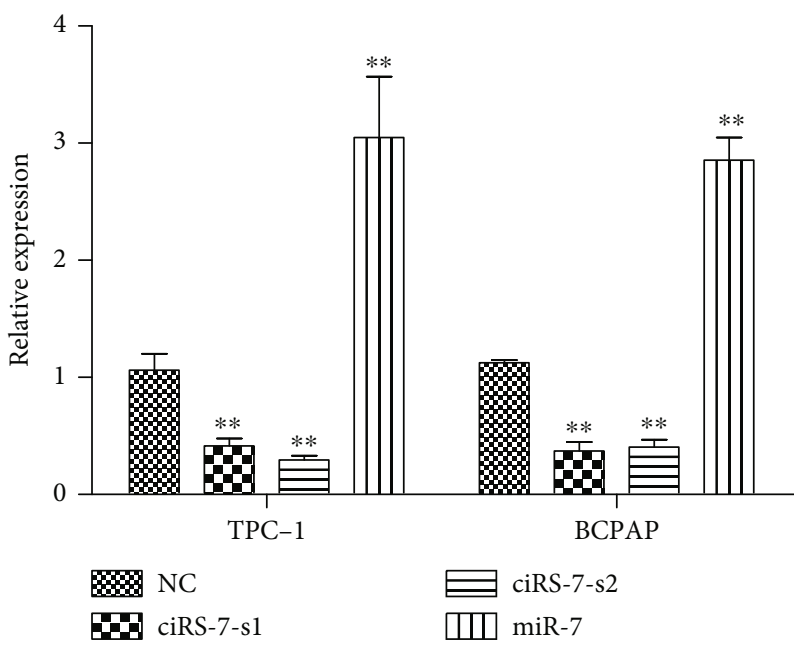

(c)

FIgURE 1: The expression of ciRS-7 in PTC. The expression of ciRS-7 in 17 pairs of matched PTC tissues and adjacent normal tissues was measured by qRT-PCR (a). The expression of ciRS-7 in PTC cell (TPC-1 and BCPAP) and human thyroid epithelial cell line Nthy-ori3-1 was measured by qRT-PCR (b). The expression of ciRS-7 was knocked down by transfection of ciRS-7-siRNA and verification by qRTPCR. The expression of miR-7 was upregulated by ciRS-7 silencing (c). ${ }^{*} P<0.05$, ${ }^{* *} P<0.01$, and ${ }^{* * *} P<0.001$.

TABLE 1: Correlation of clinicopathological features and ciRS-7 expression in PTC tissues.

\begin{tabular}{lcc}
\hline Characteristics & No. of cases & $P$ value \\
\hline Gender & 14 & \\
$\quad$ Female & 3 & 0.15 \\
$\quad$ Male & & \\
Age & 12 & 0.20 \\
$\quad<50$ & 5 & \\
$>50$ & & \\
Cancer size & 7 & 0.022 \\
$\quad<1 \mathrm{~cm}$ & 10 & \\
$>1 \mathrm{~cm}$ & & 0.015 \\
Lymph node metastasis & 5 & \\
$\quad$ Yes & 12 & \\
No & & \\
\hline
\end{tabular}

2.5. Cell Proliferation Assay and Colony Formation Assay. Cell proliferation was determined using a CCK-8 assay kit (Dojindo, Kumamoto, Japan) according to the manufacturer's instructions. Cells were seeded $\left(100 \mu \mathrm{L} ; 2 \times 10^{3}\right.$ cells per well) into 96-well plates, and CCK-8 was added at $0,24,48$, and $72 \mathrm{~h}$. Absorbance was measured at $450 \mathrm{nM}$ using an enzyme-labeling instrument (Thermo Fisher Scientific) afterward. For colony formation assay, cells were seeded (500 cells per well) into six-well plates and incubated for 10 days. The colonies were stained and observed under a microscope.

2.6. Cell Apoptosis Assay. After transfection for $48 \mathrm{~h}$, cells were collected for cell apoptosis detection. The apoptosis was measured using the PE Annexin V Apoptosis Detection Kit (BD Biosciences) according to the manufacturer's instructions. FACSCalibur was employed to detect the cell apoptosis. FACSDiva was applied to analyze the data. 
TPC-1

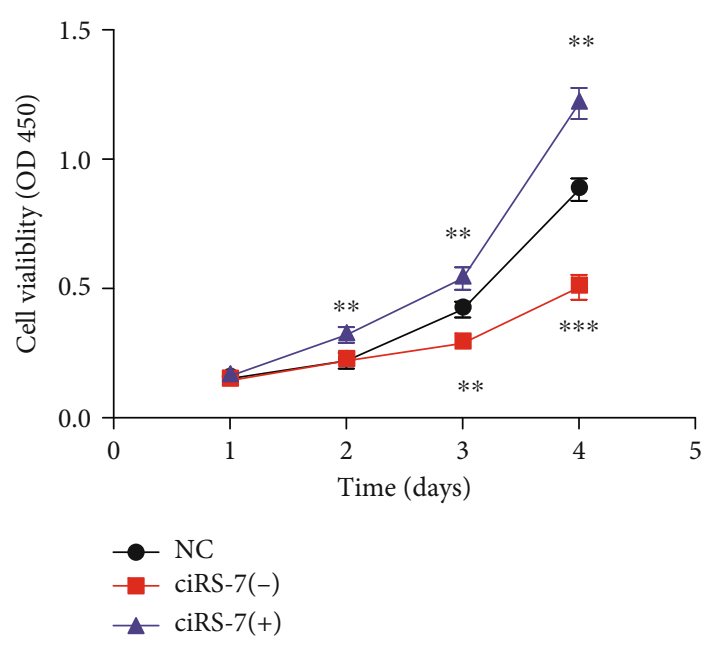

(a)

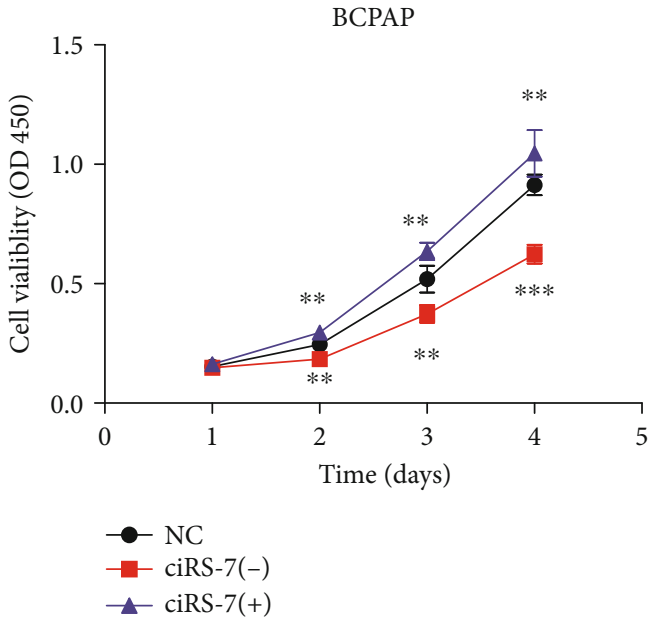

(b)

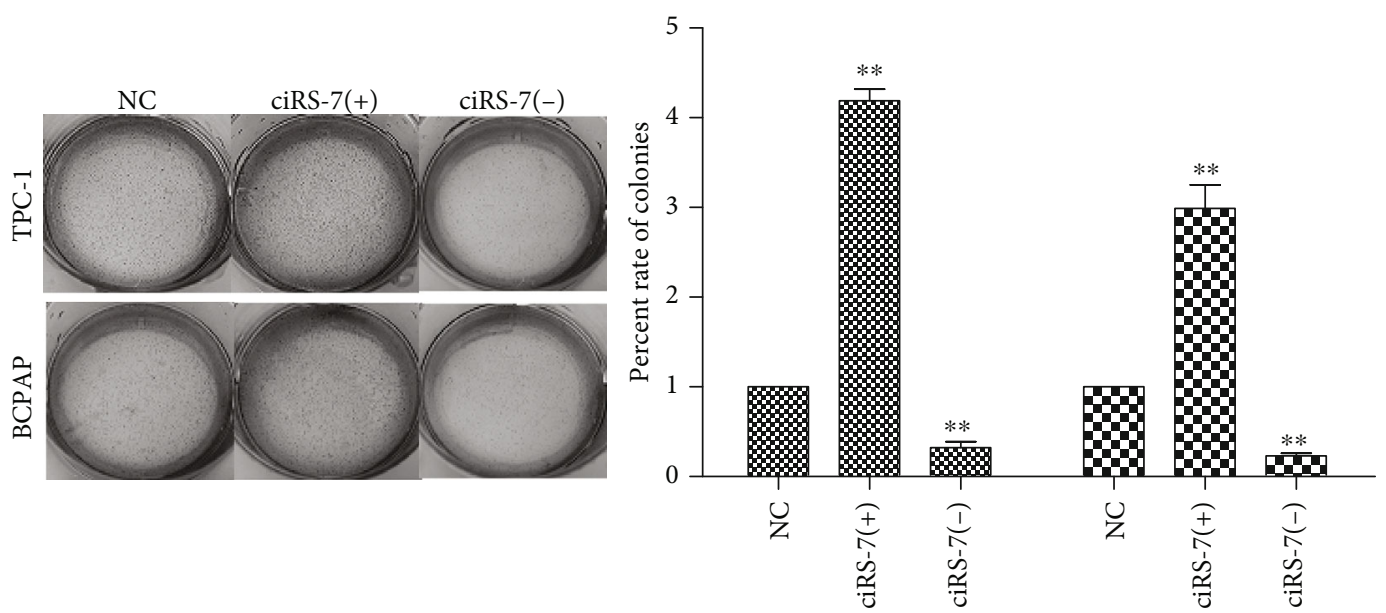

\% TPC-1

플 ВСРАР

(c)

Figure 2: Continued. 

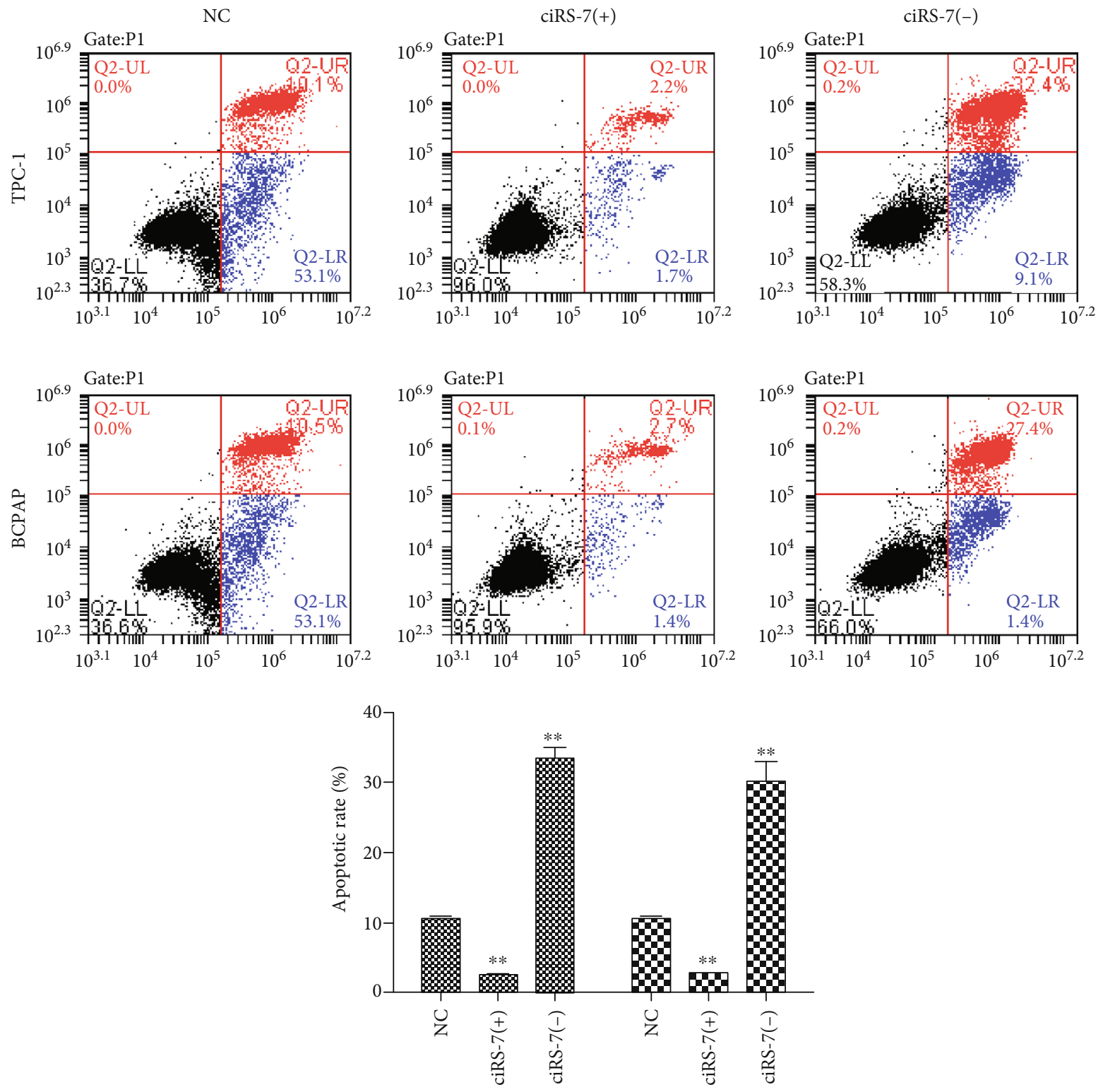

\%) TPC-1

ED BCPAP

(d)

Figure 2: The effects of ciRS-7 on PTC cell proliferation and apoptosis. Cell proliferation of PTC cells (TPC-1 and BCPAP) after ciRS-7siRNA, ciRS-7 overexpression, or negative control siRNA transfection was evaluated by CCK-8 assay (a, b). Images of colony formation assay using TPC-1 and BCPAP cells and quantification analysis of colony numbers (c). Annexin V/PI staining and FACS detection were used to detect cell apoptosis (d). ${ }^{*} P<0.05,{ }^{* *} P<0.01$, and ${ }^{* * *} P<0.001$.

2.7. Scratch Test. Cells were seeded into six-well plates to detect the wound healing capabilities. When the cells covered $80-90 \%$ of the dish, a $100 \mu \mathrm{L}$ pipette tip was used to make four scratches at the same width in each well. PBS was used to wash away the cells removed during scratching. Next, the cells were cultured in fresh culture medium in an incubator. An inverted microscope was used to observe the migration distance of cells into the scratch area at $0,6,24$, and $36 \mathrm{~h}$. The assay was repeated three times.
2.8. Transwell Migration and Invasion. Transwell migration assays were performed using a transwell chamber (Corning, China). Transfected cells in serum-free medium were seeded in the upper transwell chamber, and $500 \mu \mathrm{L}$ RPMI solution that included $20 \%$ fetal bovine (FBS) serum was added into the lower transwell chamber. After being cultured for 24 hours, cells were fixed with paraformaldehyde and stained using crystal violet. An inverted light microscope was used to quantify cell migration, and views were randomly 

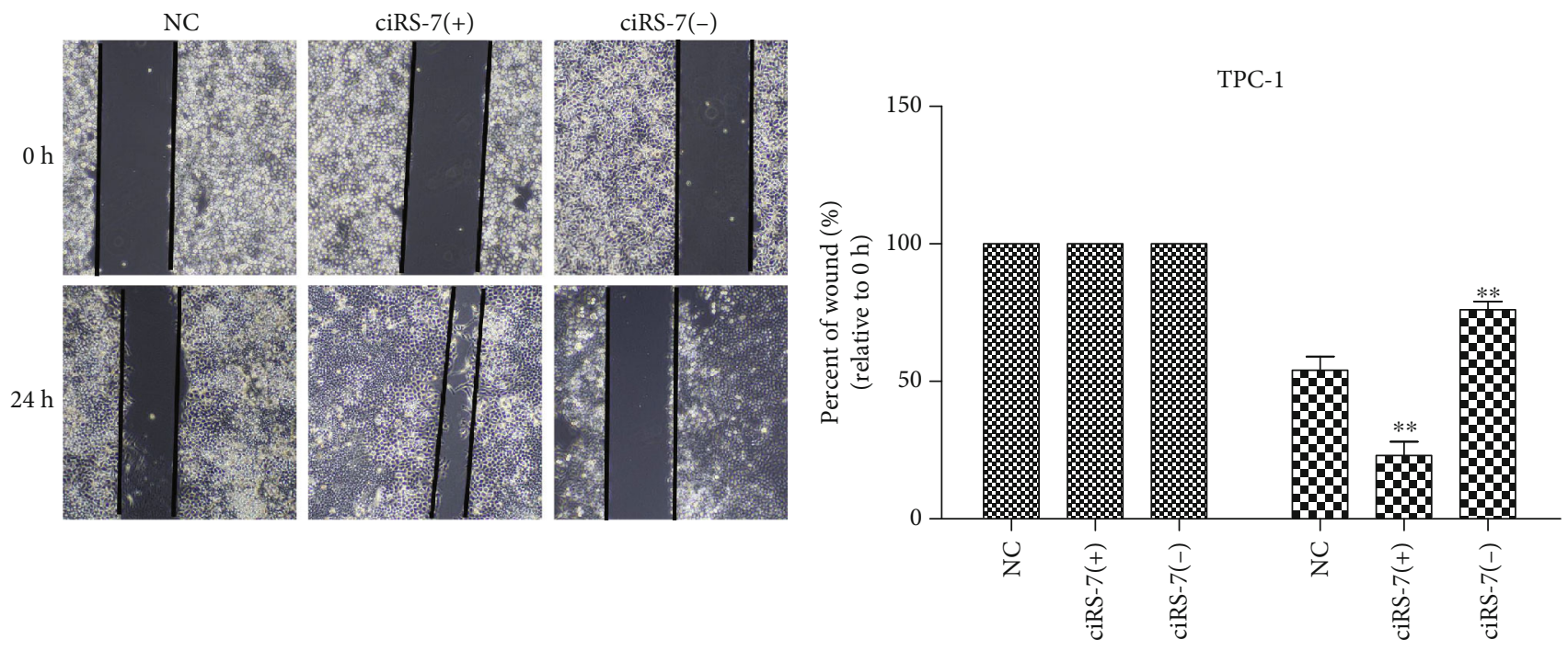

80

표 24

(a)
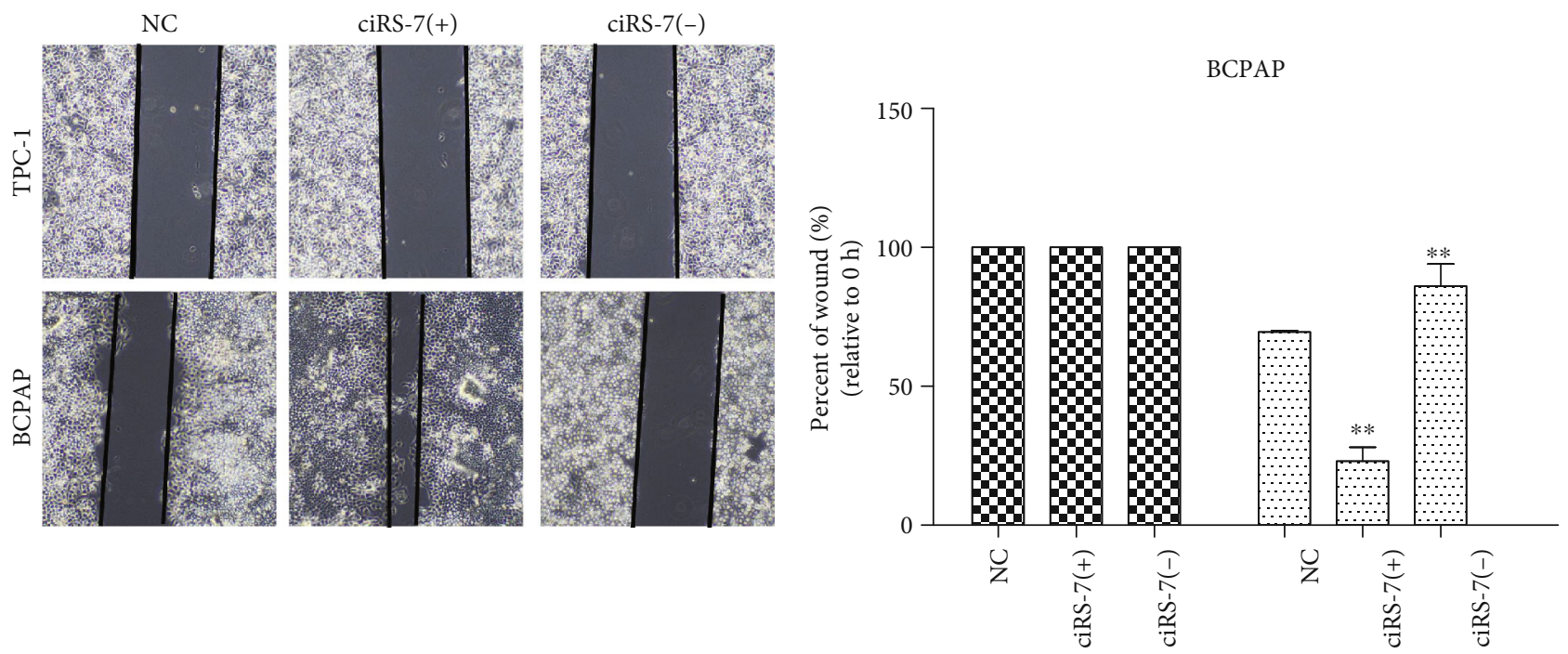

80

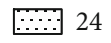

(b)

Figure 3: Continued. 

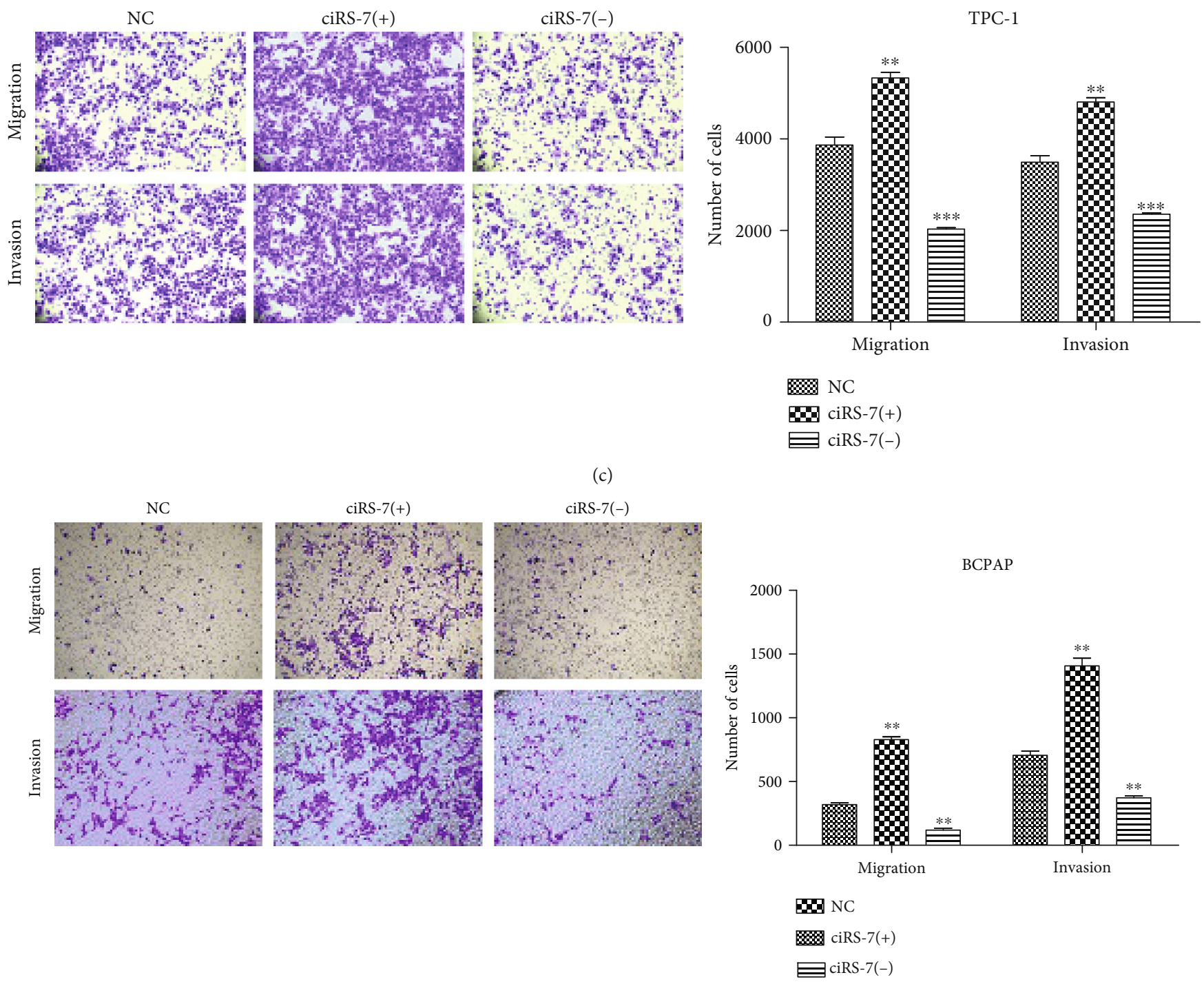

(d)

Figure 3: The effects of ciRS-7 on PTC cell migration and invasion. Wound healing assay was conducted to assess the cell migration after ciRS-7-siRNA, ciRS-7 overexpression, or negative control siRNA transfection $(\mathrm{a}, \mathrm{b})$. Transwell assays were conducted to assess the cell migration and invasion abilities of PTC cells (TPC- 1 and BCPAP) after transfection (c, d). ${ }^{*} P<0.05,{ }^{* *} P<0.01$, and ${ }^{* * *} P<0.001$.

observed to obtain the average results. Invasion assay was conducted in accordance with the above procedures except that the bottom membranes were coated with the diluted Matrigel.

2.9. Dual-Luciferase Reporter Assay. A dual-luciferase reporter assay was performed in stable PTC cells. Wild-type (WT-EGFR) and mutant (MUT-EGFR) plasmids were constructed by Genema (Shanghai, China). Four groups including microRNA-7/EGFR-WT-3'UTR, NC/EGFR-WT-3' UTR, microRNA-7/EGFR-MUT-3'UTR, and NC/EGFRMUT-3'UTR were established. After transfection for $48 \mathrm{~h}$, cells were collected for dual-luciferase activity assay using a Promega Dual-Luciferase Reporter Assay System (Promega, Madison, WI, USA).
2.10. Statistical Analysis. All statistical analyses were performed using SPSS 20.0 software (SPSS, Inc., Chicago, IL, USA). Significant differences between groups were estimated using a two-tailed Student's $t$-test, or the Wilcoxon test, as appropriate. Variables with a $P<0.05$ in univariate analyses were subsequently used for multivariate analyses based on Cox regression analyses. Two-tailed $P$ values were calculated, and statistical significance was set at $P<0.05$.

\section{Results}

3.1. The Expression of ciRS-7 in PTC Tissues and Cell Lines. To analyze the role of ciRS-7 in PTC, we measured the expression of ciRS-7 in 17 pairs of PTC and their normal counterparts. ciRS-7 was highly expressed in PTC tissues (Figure 1(a)). ciRS-7 levels were also consistently higher 


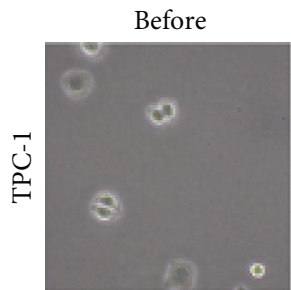

(a)

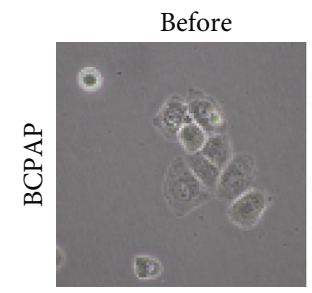

(b)

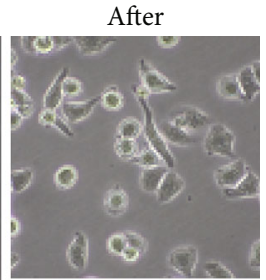

TPC-1
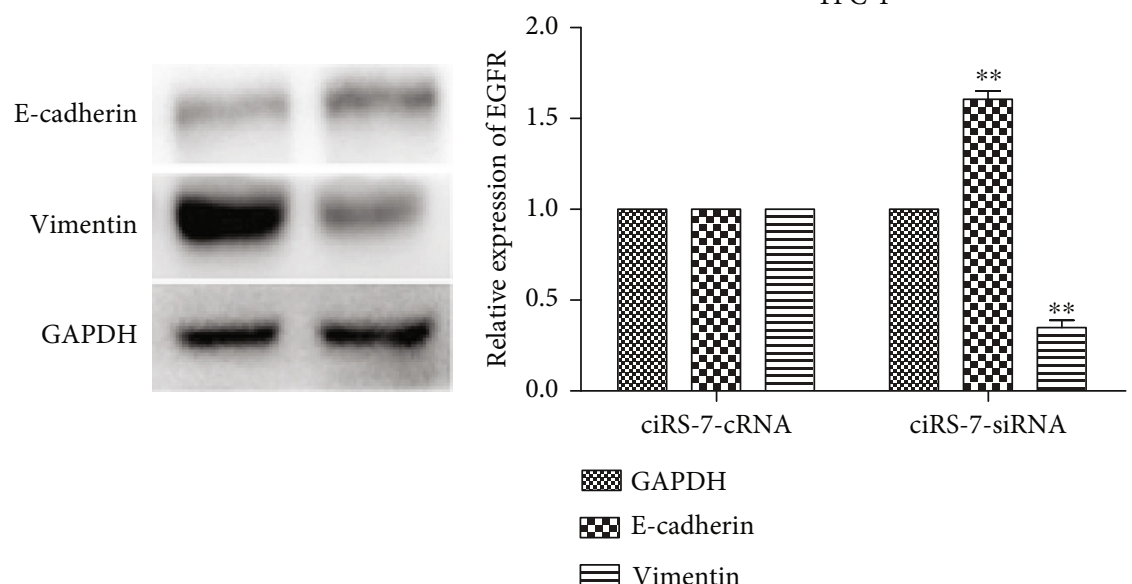

(c)
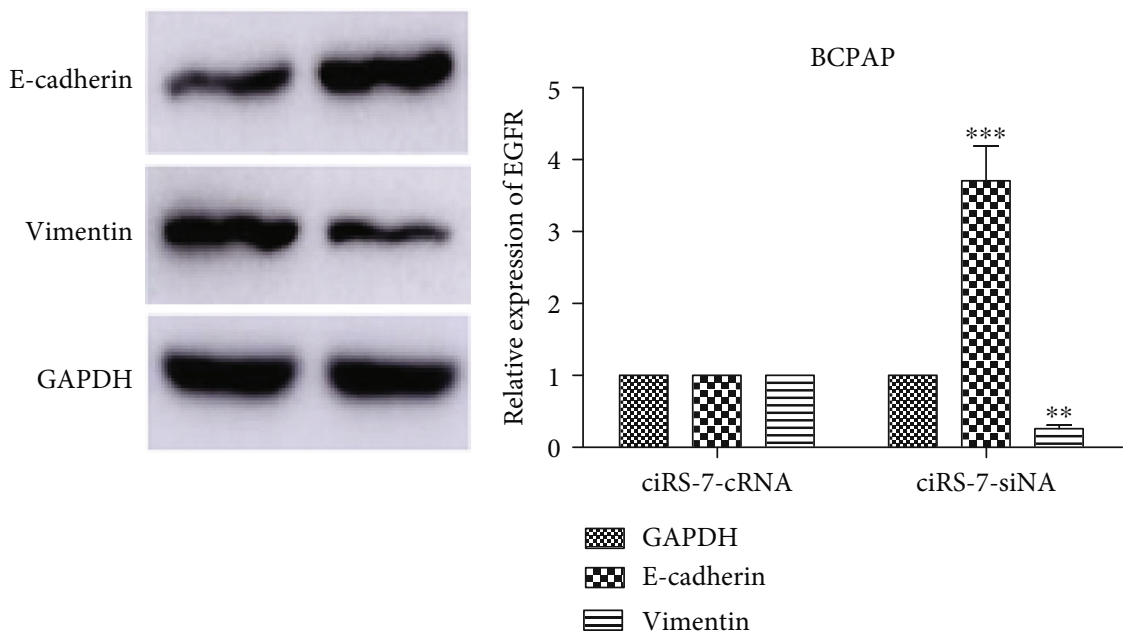

(d)

FIGURE 4: ciRS-7 silencing reduces EMT in TPC-1 cells. Cell lines exhibited a spindle-like, fibroblastic cell morphology after ciRS-7 silencing ( $\mathrm{a}, \mathrm{b})$. Western blotting assay was conducted to analyze the expression of E-cadherin and vimentin $(\mathrm{c}, \mathrm{d})$. ${ }^{*} P<0.05$, ${ }^{* *} P<0.01$, and ${ }^{* * *} P<0.001$.

in PTC cell lines TPC-1 and BCPAP than human thyroid epithelial cell line Nthy-ori 3-1 (Figure 1(b)). In addition, while the overexpression of ciRS-7 was significantly correlated with large tumor size $(P=0.015)$ and lymph metastasis $(P=0.022)$, it was not correlated with either age $(P \geq 0.05)$ or gender $(P \geq 0.05)$ (Table 1$)$.

3.2. Promotive Effects of ciRS-7 in PTC. To explore the functions of ciRS-7, two short interfering (ciRS-7-s1 and ciRS-7s2) (Supplementary Materials (available here)) vectors were transfected into TPC-1 and BCPAP cell lines (Figure 1(c)). CCK-8 assay showed that ciRS-7 downexpression significantly inhibits the in vitro proliferation of TPC-1 and BCPAP (Figures 2(a) and 2(b)). This was confirmed by colony formation assay (Figure 2(c)) and flow cytometry (Figure 2(d)). In addition, decreased invasion and migration in vitro were observed in ciRS-7-downexpressed TPC-1 and BCPAP cells using wound healing assay (Figures 3(a) and 3(b)) and transwell assays (Figures 3(c) and 3(d)). Oppositely, overexpression of ciRS-7 promoted the proliferation, 


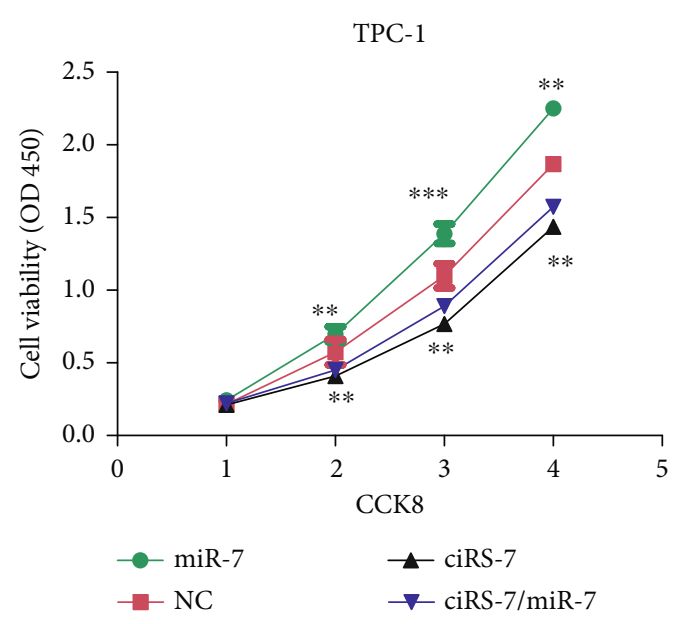

(a)

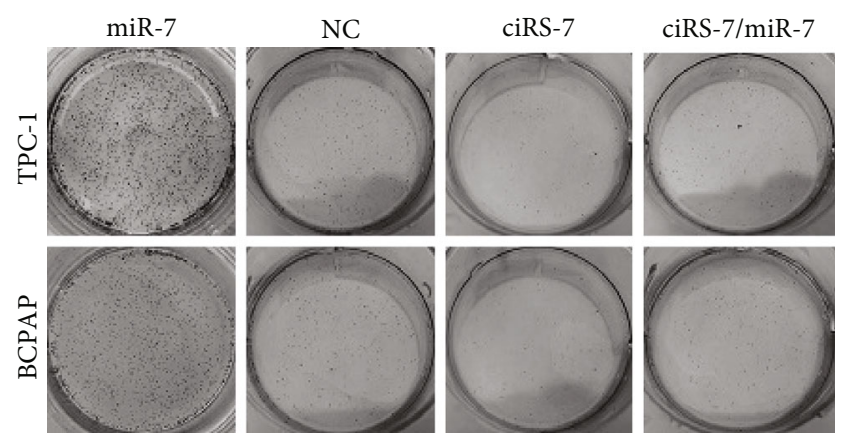

(c)
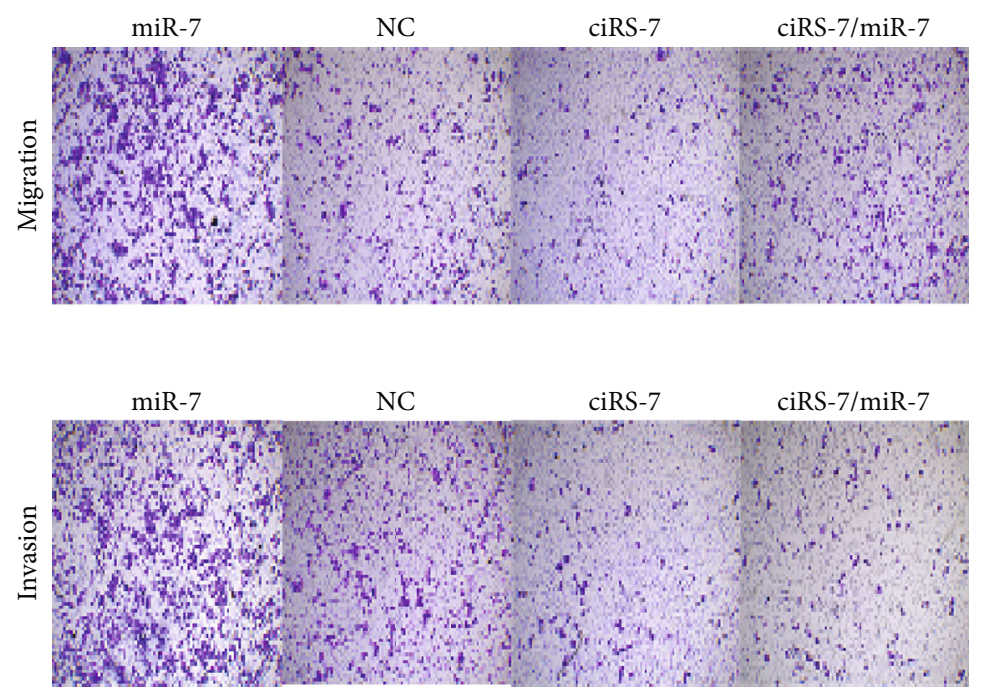

(d)

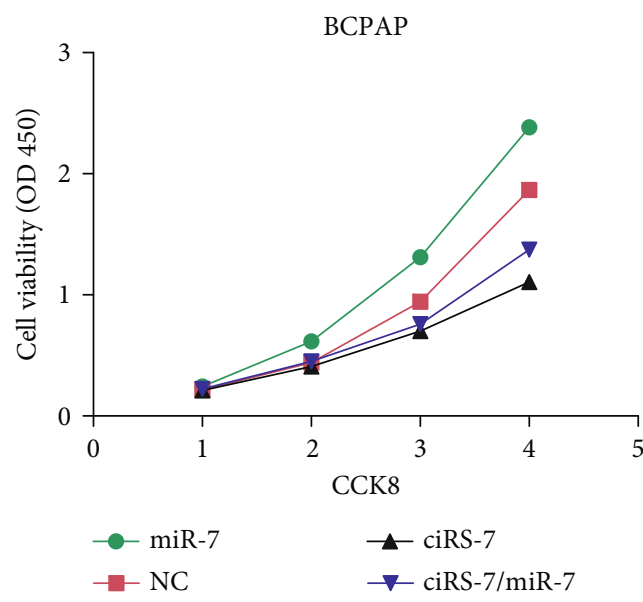

(b)

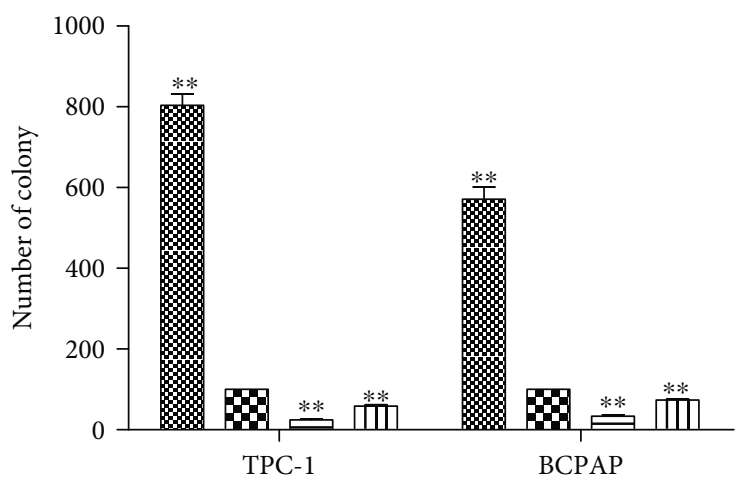

$\begin{array}{ll}2 \text { miR-7 } & \text { Ш ciRS-7/miR-7 } \\ 0 \text { NC } & \equiv \text { ciRS-7 }\end{array}$ 

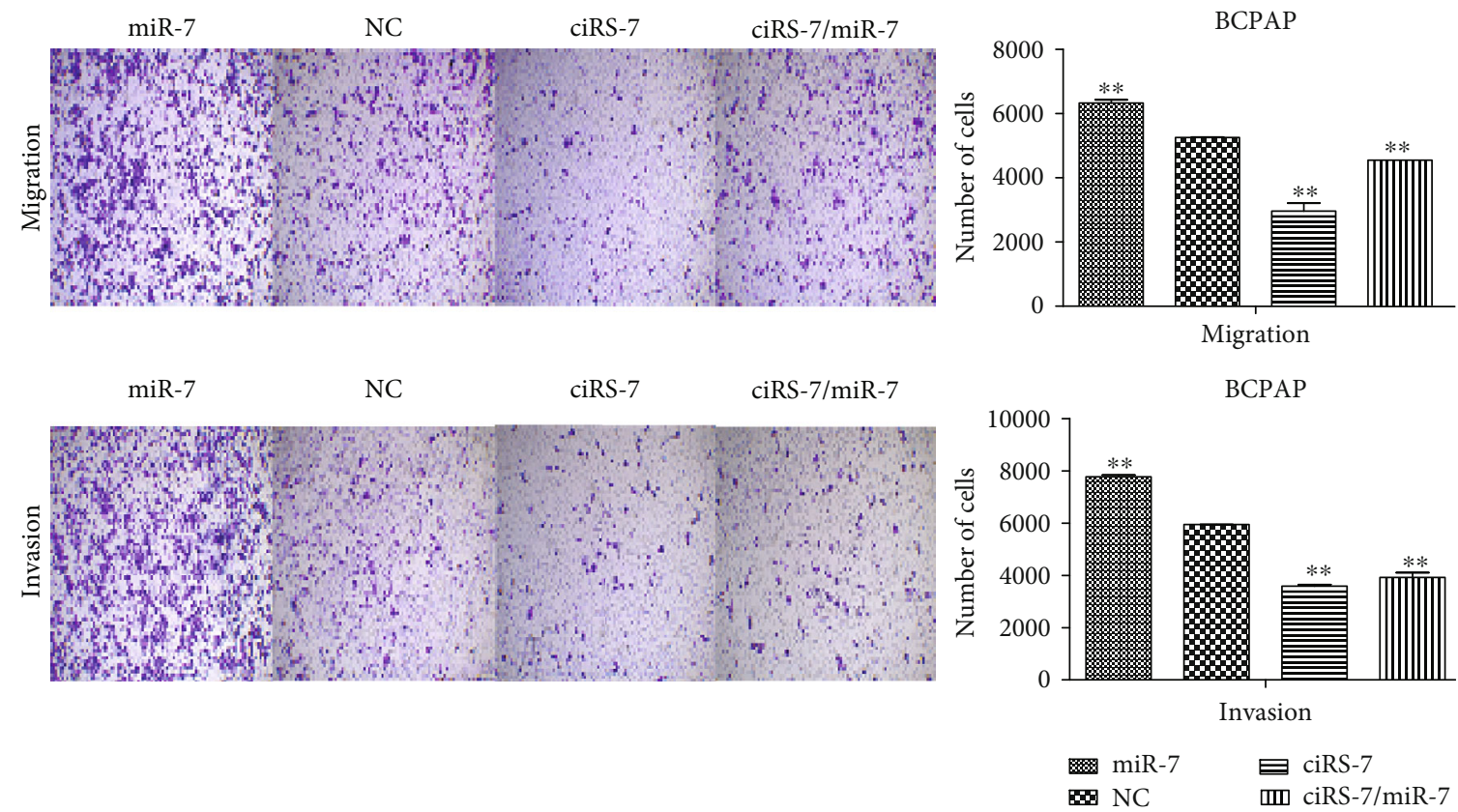

(e)

FIGURE 5: ciRS-7 silencing inhibited the promotion effect mediated by miR-7 knockdown. ciRS-7 silencing neutralized tumor cell proliferation promoted mediated by miR-7 knockdown in TPC-1 and BCPAP $(\mathrm{a}-\mathrm{c})$. ciRS-7 silencing neutralized tumor cell proliferation promoted mediated by miR-7 knockdown in TPC- 1 and BCPAP $(\mathrm{d}, \mathrm{e}) .{ }^{*} P<0.05,{ }^{* *} P<0.01$, and ${ }^{* * *} P<0.001$.

migration, and invasion in PTC cell lines (Figures 2 and 3). Collectively, the above results demonstrated that ciRS-7 plays an oncogene role in PTC.

\section{3. ciRS-7 Facilitated Epithelial-Mesenchymal Transition} (EMT) of PTC Cells. Since ciRS-7 silencing altered PTC cell morphology such that both cell lines exhibited a spindle-like, fibroblastic cell morphology (Figures 4(a) and 4(b)), we hypothesized that ciRS-7 is involved in the epithelialmesenchymal transition (EMT) of PTC cells, which is a key event associated with tumor metastasis and invasion. The western blotting results were consistent with the conclusion we expected. Vimentin levels were clearly lower in PTC cells transfected with ciRS-7-siRNA than in negative controls. Ecadherin expression was higher in the ciRS-7-siRNA group than in cells transfected with ciRS-7-cRNA (Figures 4(c) and $4(\mathrm{~d})$ ). These results indicate that downregulating ciRS7 suppresses EMT, which may explain the mechanism of invasion and migration of PTC cells.

3.4. ciRS-7 Interaction with miR-7 in PTC. Previous studies have suggested that ciRS-7 could sponge to miR-7. We evaluated the effects of ciRS-7 on regulating miR-7 in PTC cells. Our results confirmed that silencing of ciRS-7 could significantly increase the expression of miR-7 in both TPC-1 and BCPAP (Figure 1(c)). To confirm that whether ciRS-7 inhibits miR-7 for promoting oncogenic potential, CCK-8, colony formation, and transwell assays were performed in the two cell lines with knockdown of miR-7 alone, ciRS-7 alone, or both. CCK-8 and colony formation assay revealed that miR-7 knockdown increased the proliferation ability of TPC-1 and BCPAP, and such effect was partially reversed by silencing ciRS-7 (Figures 5(a)-5(d)). Consistently, ciRS-7 silencing attenuated the increased ability of migration and invasion induced by miR-7 knockdown (Figure 5(e)). Those results indicated that miR-7 is a participant in ciRS-7induced proliferation, migration, and invasion of PTC cells.

3.5. ciRS-7 Regulated EGFR by Sponging of miR-7. Bioinformatics tools (TargetScan, miRBase, and PicTar) were used to predict the potential target of microRNA-7 (Figure 6(d)). Among the overlap target genes, epidermal growth factor receptor (EGFR) was selected because of its role in cancer development. To further confirm whether miR-7 regulates the expression of EGFR directly, we constructed the wildtype and mutated $3^{\prime}$ UTR of human EGFR mRNA and cotransfected with hsa-miR-7 mimic into TPC-1. We found that miR-7 significantly reduced the luciferase intensity in the cells cotransfected with wild-type $3^{\prime}$ UTR of EGFR but not the mutant one (Figure 6(e)).

Western blotting assay showed that the expression of EGFR can be decreased by ciRS-7 silencing in TPC- 1 and BCPAP (Figure 6(a)). Therefore, we increased the expression of EGFR in PTC cell. The results revealed that overexpressed EGFR could reverse the effects of ciRS-7 silencing on proliferation (Figure 6(b)), migration, and invasion (Figure 6(c)) in vitro. The consistent results suggested that EGFR could be involved in the promotion effects of ciRS-7/miR-7 on PTC cells.

\section{Discussion}

circRNAs have been reported to be participants in the development of various cancers including thyroid cancers. As one of the most extensively investigated circRNA, ciRS-7 is 

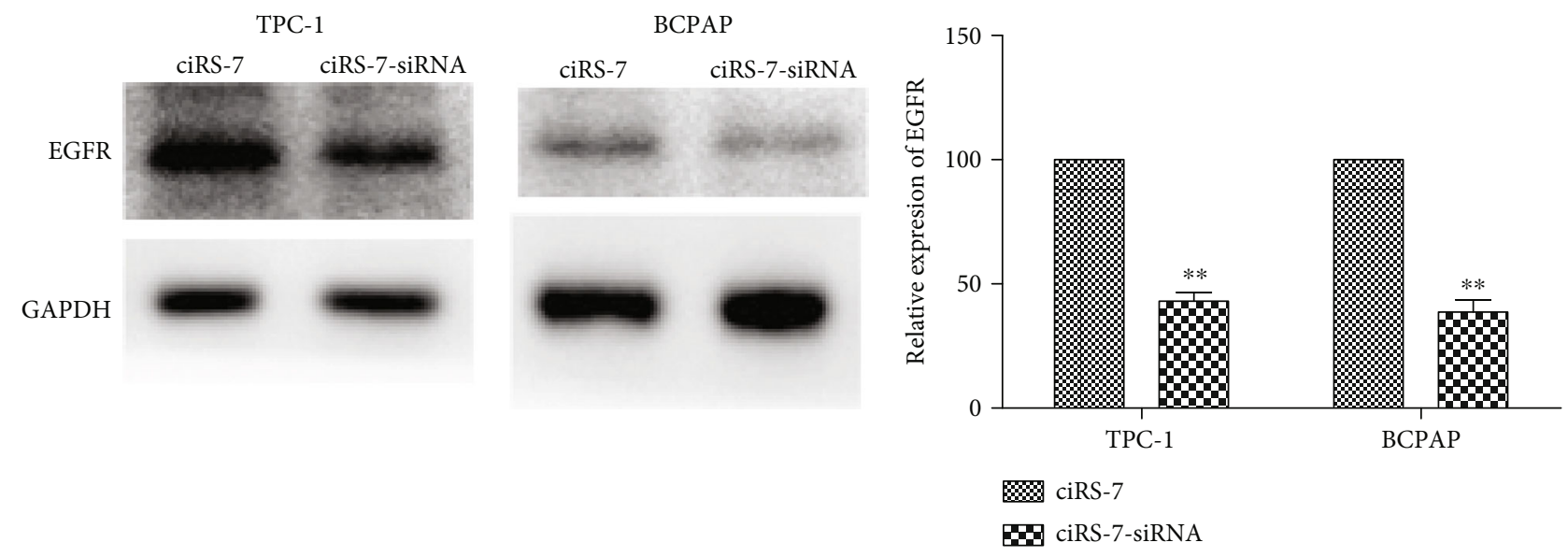

(a)
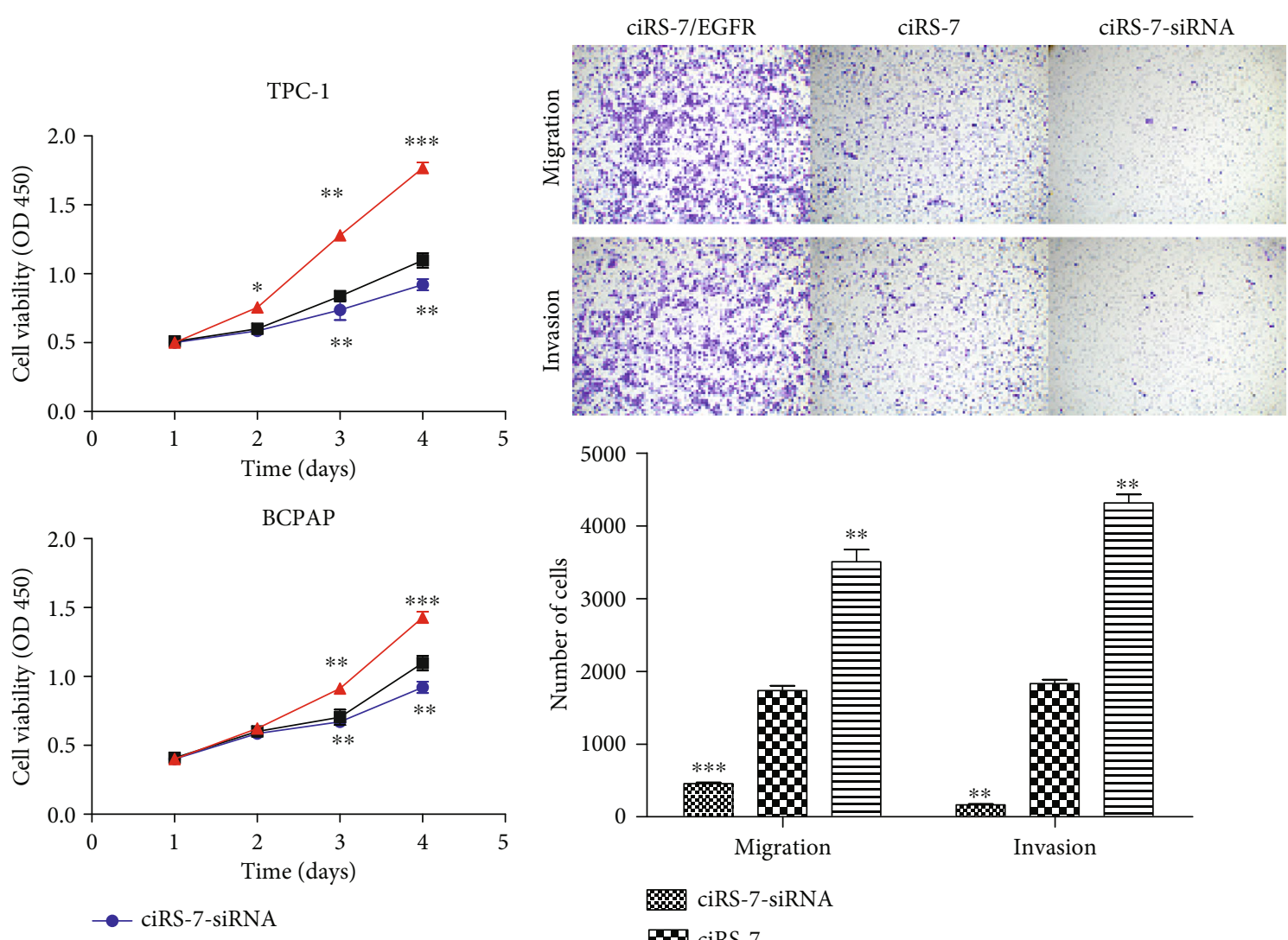

\% ciRS-7-siRNA

Ad ciRS-7

$\rightarrow$ - ciRS-7

$\triangle$ ciRS-7/EGFR

ciRS-7/EGFR

(b)

(c)

Figure 6: Continued. 


\begin{tabular}{|c|c|}
\hline EGFR wt URT & 5'...GAGCACAAGCCACAA GUCUUCCA.. \\
\hline miR-7 & 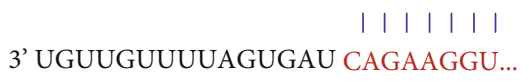 \\
\hline
\end{tabular}

(d)

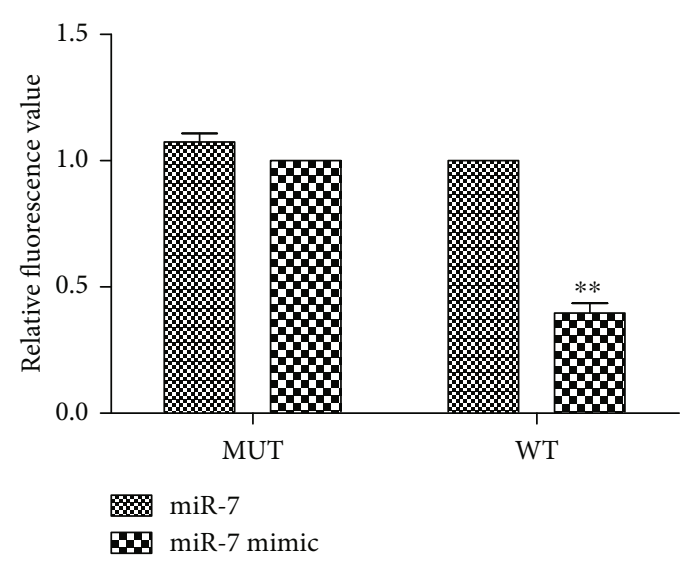

(e)

FIGURE 6: EGFR mediated the promotion effects of ciRS-7/miR-7 axis on PTC cells. The expression of EGFR was decreased by ciRS-7 silencing in TPC-1 and BCPAP (a). Overexpressed EGFR could reverse the effects of ciRS-7 silencing on proliferation, migration, and invasion $(b, c)$. The conserved binding site in $3^{\prime}$ UTR of human EGFR mRNA to miR-7 (d). Luciferase system analysis was used to identify the direct binding of miR-7 to $3^{\prime}$ UTR of EGFR (e). ${ }^{*} P<0.05,{ }^{* *} P<0.01$, and ${ }^{* * *} P<0.001$.

known to modulate proliferation, invasion, and migration in cancers including hepatocellular carcinoma, colorectal, gastric, and lung cancers [14-16]. Experimental data indicated that it could be a valuable biomarker and therapeutic target in esophageal squamous cell carcinoma (ESCC) [17]. In this study, we investigated for the first time the function and mechanism of ciRS-7 in PTC. Our qRT-PCR results showed that ciRS-7 expression was higher in PTC tissues than in normal samples. Overexpressed ciRS-7 contributes to the aggressive clinicalpathological factors such as large tumor size and lymph node metastasis. Moreover, ciRS-7 silencing significantly inhibited PTC proliferation, migration, and invasion in vitro. Overexpressed ciRS-7 promoted PTC proliferation, migration, and invasion. Together with the consistent clinical data, the oncogenic role of ciRS-7 in PTC was significantly supported.

Next, we explored the mechanism by which ciRS-7 affects the development of PTC. After ciRS-7 silencing, PTC cells transformed into spindle epithelium morphologically. We hypothesized that ciRS-7 is involved in the epithelialmesenchymal transition (EMT) of PTC cells, which was activated during cancer and promoted migration and invasion [18]. We detected the EMT biomarker E-cadherin and vimentin; the level of E-cadherin was higher while vimentin was lower when ciRS-7 was decreased after ciRS-7 silencing. EMT was inhibited by ciRS-7 silencing, which could partly explain how ciRS-7 silencing inhibited migration and invasion in PTC.

Our present study further revealed that ciRS-7 triggered progression of PTC cells via regulating the miR-7/EGFR axis. ciRS-7 contains 74 binding sites for miR-7 and binds densely to it as an inhibitor. Previously, ciRS-7 has been shown to block miR-7 and thereby reactivate genes suppressed by miR-7 in the brain [19], islet cells [20], colorectal cancer [21], lung cancer, and gastric cancer (GC) [22]. miR-7 has been reported to be a tumor suppressor in various tumors. In PTC, miR-7 suppresses cell growth and development via downregulation of many oncogenic signaling pathway such as CKS2/cyclin B2 and cdk1, p21-activated kinase-1 (PAK1). Our data provided another potential downstream target of miR-7 when it is involved in proliferation, migration, and invasion in PTC.

EGFR is a member of the HER family of receptors and is a receptor for members of the EGF family [23]. During tumor progression, specific endogenous ligands activate downstream pathways including the Ras/Raf mitogen-activated protein kinase (MAPK), Jak2/Stat3, and PI3K/AKT pathway [24]. The EGFR/RAF/MAPK pathway is a well-known oncogenic pathway which correlates with metastasis [25]. The JAK/STAT3 signaling could promote invasion and metastasis through activation of key metastasis-promoting genes such as WASF3 [26]. The PI3K/AKT signaling pathway is a crucial player in the regulation of different cellular and molecular processes including cell growth, proliferation, cell motility, and survival in PTC [27]. Furthermore, its therapeutic potential in human cancers has been addressed. In lung cancer, EGFR has become a crucial therapeutic target for patients with non-small-cell lung cancer. EGFR tyrosine kinase inhibitors are the most promising clinical agents as monotherapy for non-small-cell lung cancer [28]. Additionally, data suggest that blocking EGFR can effectively increase the antitumor activity of selumetinib in triple-negative breast cancer, which may be related to the effect of this combination on the activation of extracellular signal-regulated kinase 1/2 and AKT [29]. In PTC, EGFR was demonstrated to upregulate in about $55 \%$ of PTCs and is correlated with aggressive behaviors of PTC [30].

Using publicly available algorithms (TargetScan, miRanda, and PicTar), we identified EGFR as a potential target of miR-7. Luciferase reporter assay further demonstrated their interaction. Moreover, we found that the protein level of EGFR is decreased distinctly in ciRS-7-silencing cells. EGFR overexpression significantly reversed the effects of ciRS-7 silencing on PTC cell proliferation, migration, and invasion, indicating that EGFR was involved in the progression induced by the ciRS-7/miR-7 axis in PTC. We believed that ciR-7 inhibited proliferation, migration, and invasion of PTC by targeting the miR-7/EGFR axis. 


\section{Conclusion}

Our results indicated that ciRS-7 promotes the genesis and development of PTC by increasing the proliferation and migration of PTC cells. Regulation of the ciRS-7/miR7/EGFR axis is a crucial molecular mechanism in PTC, and this pathway may be a novel target for the diagnosis and therapy of this cancer.

\section{Data Availability}

The raw data required to reproduce these findings cannot be shared at this time as the data also forms part of an ongoing study.

\section{Ethical Approval}

All procedures involving human participants were in accordance with the ethical standards of the institutional and/or national research committee and with the 1964 Helsinki Declaration and its later amendments or comparable ethical standards.

\section{Consent}

All patients provided informed written consent for all procedures in the study.

\section{Conflicts of Interest}

The authors declare no conflict of interest.

\section{Authors' Contributions}

Jun-ya Han, Jingjing $\mathrm{Xu}$, and Kuisheng Chen designed the study; Rui Xue, Wencai Li, Gang Dong, and Jianhua Li collected patients' data. Jun-ya Han and Kuisheng Chen wrote the manuscript, and Jingjing $\mathrm{Xu}$ revised and approved the version to be submitted. Si Guo contributed significantly to the analysis and manuscript preparation. Na Wei, Xiangyu Tian, and Chao Chen contributed to experimental studies and manuscript review. Sen Qiu, Tong Wang, and Qiankun Xiao contributed to clinical studies.

\section{Acknowledgments}

We are grateful to Henan Key Laboratory of Tumor Pathology, the First Affiliated Hospital of Zhengzhou University, Zhengzhou, China. We also thank the members of the Department of Pathology, the First Affiliated Hospital of Zhengzhou University, Zhengzhou, China. We also thank all patients for providing consent to participate in the study. This work was supported by the National Natural Science Foundation of China (81600944), the Henan Provincial Department of Science and Technology Research (182102310512), and the Henan medical science and technology project jointly built by the ministry and the province (SB201902003).

\section{Supplementary Materials}

To explore the functions of ciRS-7, two short interfering (ciRS-7-s1 and ciRS-7-s2) vectors were transfected into TPC-1 cell line. The effects of ciRS-7 silencing on PTC is shown. (Supplementary Materials)

\section{References}

[1] D. Eng, "Management guidelines for motor neurone disease patients on non-invasive ventilation at home," Palliative Medicine, vol. 20, no. 2, pp. 69-79, 2016.

[2] N. Kurtulmus, B. Ertas, Y. Saglican, H. Kaya, U. Ince, and M. Duren, "BRAFV600E mutation: has it a role in cervical lymph node metastasis of papillary thyroid cancer?," European Thyroid Journal, vol. 5, no. 3, pp. 195-200, 2016.

[3] C. K. Zhao, J. Y. Zheng, L. P. Sun, R. Y. Xu, Q. Wei, and H. X. $\mathrm{Xu}$, "BRAF ${ }^{\mathrm{V} 600 \mathrm{E}}$ mutation analysis in fine-needle aspiration cytology specimens for diagnosis of thyroid nodules: the influence of false-positive and false-negative results," Cancer Medicine, vol. 8, no. 12, pp. 5577-5589, 2019.

[4] P. Agretti, F. Niccolai, T. Rago et al., "BRAF mutation analysis in thyroid nodules with indeterminate cytology: our experience on surgical management of patients with thyroid nodules from an area of borderline iodine deficiency," Journal of Endocrinological Investigation, vol. 37, no. 10, pp. 1009-1014, 2014.

[5] Y. Ito, A. Miyauchi, M. Kihara, M. Fukushima, T. Higashiyama, and A. Miya, "Overall survival of papillary thyroid carcinoma patients: a single-institution long-term follow-up of 5897 patients," World Journal of Surgery, vol. 42, no. 3, pp. 615-622, 2018.

[6] A. Doubleday and R. S. Sippel, "Surgical options for thyroid cancer and post-surgical management," Expert Review of Endocrinology and Metabolism, vol. 13, no. 3, pp. 137-148, 2018.

[7] P. Fallahi, I. Ruffilli, G. Elia et al., "Novel treatment options for anaplastic thyroid cancer," Expert Review of Endocrinology and Metabolism, vol. 12, no. 4, pp. 279-288, 2017.

[8] Z. J. Zhao and J. Shen, "Circular RNA participates in the carcinogenesis and the malignant behavior of cancer," RNA Biology, vol. 14, no. 5, pp. 514-521, 2016.

[9] B. Chen and S. Huang, "Circular RNA: an emerging noncoding RNA as a regulator and biomarker in cancer," Cancer Letters, vol. 418, pp. 41-50, 2018.

[10] R. Li, B. Wu, J. Xia, L. Ye, and X. Yang, "Circular RNA hsa_circRNA_102958 promotes tumorigenesis of colorectal cancer via miR-585/CDC25B axis," Cancer Management and Research, vol. 11, pp. 6887-6893, 2019.

[11] J. Song, H. L. Wang, K. H. Song et al., “CircularRNA_104670 plays a critical role in intervertebral disc degeneration by functioning as a ceRNA," Experimental \& Molecular Medicine, vol. 50, no. 8, p. 94, 2018.

[12] X. B. Zheng, M. Zhang, and M. Q. Xu, "Detection and characterization of ciRS-7: a potential promoter of the development of cancer," Neoplasma, vol. 64, no. 3, pp. 321-328, 2017.

[13] X. Zhou, L. Jiang, G. Fan et al., "Role of the ciRS-7/miR-7 axis in the regulation of proliferation, apoptosis and inflammation of chondrocytes induced by IL- $1 \beta$," International Immunopharmacology, vol. 71, pp. 233-240, 2019.

[14] L. Xu, M. Zhang, X. Zheng, P. Yi, C. Lan, and M. Xu, “The circular RNA ciRS-7 (Cdrlas) acts as a risk factor of hepatic microvascular invasion in hepatocellular carcinoma," Journal 
of Cancer Research and Clinical Oncology, vol. 143, no. 1, pp. 17-27, 2017.

[15] B. Yan, W. Zhang, X. W. Mao, and L. Y. Jiang, "Circular RNA ciRS-7 correlates with advance disease and poor prognosis, and its down-regulation inhibits cells proliferation while induces cells apoptosis in non-small cell lung cancer," European Review for Medical and Pharmacological Sciences, vol. 22, no. 24, pp. 8712-8721, 2018.

[16] C. Su, Y. Han, H. Zhang et al., "CiRS-7 targeting miR-7 modulates the progression of non-small cell lung cancer in a manner dependent on NF- $\kappa \mathrm{B}$ signalling," Journal of Cellular and Molecular Medicine, vol. 22, no. 6, pp. 3097-3107, 2018.

[17] M. Sang, L. Meng, Y. Sang et al., "Circular RNA ciRS-7 accelerates ESCC progression through acting as a miR-876-5p sponge to enhance MAGE-A family expression," Cancer Letters, vol. 426, pp. 37-46, 2018.

[18] C. L. Chaffer, J. B. San, E. Lim, and R. A. Weinberg, "EMT, cell plasticity and metastasis," Cancer Metastasis Reviews, vol. 35, no. 4, pp. 645-654, 2016.

[19] D. Lu and A. D. Xu, "Mini review: circular RNAs as potential clinical biomarkers for disorders in the central nervous system," Frontiers in Genetics, vol. 7, p. 53, 2016.

[20] H. Xu, S. Guo, W. Li, and P. Yu, “The circular RNA Cdr1as, via miR-7 and its targets, regulates insulin transcription and secretion in islet cells," Scientific Reports, vol. 5, no. 1, 2015.

[21] W. Weng, Q. Wei, S. Toden et al., "Circular RNA ciRS-7-A Promising Prognostic Biomarker and a Potential Therapeutic Target in Colorectal Cancer," Clinical Cancer Research, vol. 23, no. 14, pp. 3918-3928, 2017.

[22] H. Pan, T. Li, Y. Jiang et al., "Overexpression of circular RNA ciRS-7 abrogates the tumor suppressive effect of miR-7 on gastric cancer via PTEN/PI3K/AKT signaling pathway," Journal of Cellular Biochemistry, vol. 119, no. 1, pp. 440-446, 2018.

[23] W. Cheng, J. Zhou, X. Tian, and X. Zhang, "Development of the third generation EGFR tyrosine kinase inhibitors for anticancer therapy," Current Medicinal Chemistry, vol. 23, no. 29, pp. 3343-3359, 2016.

[24] S. Bogdan and C. Klambt, "Epidermal growth factor receptor signaling," Current Biology, vol. 11, no. 8, pp. R292-R295, 2001.

[25] Y. Sun, W. Z. Liu, T. Liu, X. Feng, N. Yang, and H. F. Zhou, "Signaling pathway of MAPK/ERK in cell proliferation, differentiation, migration, senescence and apoptosis," Journal of Receptor and Signal Transduction Research, vol. 35, no. 6, pp. 600-604, 2015.

[26] Y. Teng, J. L. Ross, and J. K. Cowell, "The involvement of JAKSTAT3 in cell motility, invasion, and metastasis," JAK-STAT, vol. 3, no. 1, article e28086, 2014.

[27] Z. Nozhat and M. Hedayati, "PI3K/AKT pathway and its mediators in thyroid carcinomas," Molecular Diagnosis \& Therapy, vol. 20, no. 1, pp. 13-26, 2016.

[28] S. M. Lim, N. L. Syn, B. C. Cho, and R. A. Soo, "Acquired resistance to EGFR targeted therapy in non-small cell lung cancer: mechanisms and therapeutic strategies," Cancer Treatment Reviews, vol. 65, pp. 1-10, 2018.

[29] Z. Wang, "ErbB receptors and cancer," Methods in Molecular Biology, vol. 1652, pp. 3-35, 2017.

[30] Y. J. Dai, Y. B. Qiu, R. Jiang et al., "Concomitant high expression of ER $\alpha 36$, EGFR and HER2 is associated with aggressive behaviors of papillary thyroid carcinomas," Scientific Reports, vol. 7, no. 1, article 12279, 2017. 